\title{
Exploring the Role of the Microbiome in Multiple Sclerosis
}

\author{
Lloyd Kasper, ${ }^{1}$ Tanuja Chitnis, ${ }^{2,3}$ Mirla Avila, ${ }^{4}$ Nicole Sconzo, ${ }^{5}$ Raymond Ashton ${ }^{6}$ and Mark Rametta \\ 1. Departments of Microbiology/Immunology and Medicine, Geisel School of Medicine, Dartmouth College, Hanover, NH, USA; 2. Harvard Medical \\ School, Partners Pediatric Multiple Sclerosis Center, Massachusetts General Hospital, Boston, MA, USA; 3. Translational Neuroimmunology Research \\ Center and Partners Multiple Sclerosis Center, Brigham and Women's Hospital, Boston, MA, USA; 4. Multiple Sclerosis and Demyelinating Diseases \\ Clinic, Department of Neurology, Texas Tech University Health Science Center, Lubbock, TX, USA; 5. Clinical Nutrition-Private Practice, Sparta, NJ, USA; \\ 6. Ashton Medicom, Salfords, Surrey, UK; 7. US Medical Affairs, Neurology, Bayer HealthCare Pharmaceuticals Inc., Whippany, NJ, USA
}

DOI: https://doi.org/10.17925/USN.2019.15.2.82

$\mathrm{M}$ ultiple sclerosis (MS) is considered to be an immune-mediated disease with both environmental factors and genetics involved in its etiology. In the human body, most mucosal surfaces are colonized by microbiota, with the gut being the largest and probably most important in terms of the homeostatic balance of the immune system. Over 100 trillion bacteria, viruses, archaea, and fungi comprise the gut microflora, which is unique in all living organisms, outnumber human cells by at least 10-fold and encode over 100-fold more unique genes than the host genome. The interaction of the host genome with the extensive genome of the microflora on any mucosal surface within the host defines the microbiome. In the human gut, Firmicutes and Bacteroidetes have been identified as the dominant phyla of the healthy mammalian body. Interactions between the host microbiota and the gut-associated lymphoid tissue (GALT) influence and shape the immune system locally and systemically. The GALT has become a focal point in research, since it is responsible for the activation of both immune inflammatory and regulatory cells by various bacterial strains in their respective phyla. In normal circumstances the homeostatic balance between inflammation and regulation leads to immune tolerance in the host. However, the imbalance or dysbiosis (altered gut microflora) of the gut flora can lead to a disease state that is observed in those with auto-inflammatory conditions such as MS. Dysbiosis has been observed in the experimental inflammatory and demyelinating condition, experimental autoimmune encephalomyelitis (EAE). Passive transfer of dysbiotic flora from an EAE mouse to a normal mouse results in the development of EAE. Patients with MS seem to have increases in some phyla and decreases in others. Considerable evidence has suggested that MS affects the composition of the gut microbiota with some bacterial species that promote immunosuppressive T regulatory cells being less evident. It is currently uncertain if changes in the gut microflora contribute to the pathogenesis of MS or conversely if the disease state leads to the dysbiotic imbalance. We are on the forefront of understanding for a new wave of "human biology" that may lead to a wide range of therapeutic interventions that will revitalize the dysbiotic mucosal surface in a wide range of human disease conditions including MS.

\section{Keywords}

Multiple sclerosis, microbiome, dysbiosis, probiotics, prebiotics

Disclosures: Lloyd Kasper has received funding from the National Institutes of Health $(\mathrm{NIH})$, the National MS Society; has served as an advisor and consultant to Teva NeuroScience, Genzyme/Sanofi; and is a co-founder of Symbiotix Biotech. Tanuja Chitnis has served as an advisor for Biogen-Idec, Novartis, and Alexion and serves on clinical trial advisory boards for Novartis and Sanofi Aventis. She has received research support from the Department of Defense, NIH, National MS Society, Guthy-Jackson Charitable Foundation, Verily, EMD Serono and Novartis. Mirla Avila has received consulting fees from Teva, Biogen, Genzyme, Novartis and EMD Serono for patient and physician education programs as well as advisory committees. Nicole Sconzo has nothing to declare in relation to this article. Raymond Ashton is the director of Ashton Medicom. Mark Rametta is employed by Bayer HealthCare Pharmaceuticals.

Review Process: Double-blind peer review.

Compliance with Ethics: This article involves a review of the literature and did not involve any studies with human or animal subjects performed by any of the authors.

Authorship: All named authors meet the criteria of the International Committee of Medical Journal Editors for the authorship for this manuscript, take responsibility for the integrity

of the work as a whole and have given final approval for the version to be published.

Received: March 25, 2019

Accepted: April 9, 2019

Citation: US Neurology. 2019;15(2):82-90

Corresponding Author: Lloyd Kasper, Departments of Microbiology/Immunology and Medicine, Geisel School of Medicine, Dartmouth College, Hanover, NH 03755, USA. E: lloyddartmouth@gmail.com

Support: The publication of this article was supported by Bayer HealthCare Pharmaceuticals, who were given the opportunity to review the article for scientific accuracy before submission. Any resulting changes were made at the authors' discretion.
Multiple sclerosis (MS) involves inflammation, demyelination and neurodegeneration of the central nervous system (CNS) and is characterized by variable degrees of axonal loss and gliosis. ' Lesions occur in both the white and grey matter. Generally, the overall incidence of MS and other chronic autoimmune disorders has increased in the last 100 years. ${ }^{2}$ The incidence and prevalence of MS varies worldwide with genetic and geographical factors, but overall prevalence has been rising as exemplified by data from British Columbia, Canada. Prevalence increased by $4.7 \%$ on average per year between 1991-2008 (78.8/100,000 versus 179.9 respectively; $p<0.001$ ), although incidence rate was stable. ${ }^{3}$ It is possible that changes in eating habits may be responsible for dysbiosis (altered gut microflora) and dysregulation in the immune network, leading to an increase in patients with MS. In addition, there is generally a reduction in infectious diseases leading to an increase in autoimmunity, which could be due to a cleaner immediate environment experienced in the last decades.

MS is considered to be an immune-mediated disease with both environmental factors and genetics involved in its etiology. ${ }^{1,45}$ Epidemiological investigations have identified a number of causal and risk factors including vitamin D insufficiency, smoking, obesity and diet, geographic location, stress, genetics, and possibly Epstein-Barr virus infection..$^{1,6}$ The role of the environment is supported by numerous studies of migration reported in the last few decades. ${ }^{1,2,4}$ However, although disease incidence in these studies was linked to the location of the patient, this has now become less 
apparent due to increasing globalization. ${ }^{4}$ Furthermore, during the latter part of the $19^{\text {th }}$ century, many people have left their rural agricultural way of life in exchange for more industrialized existence in towns and cities, which resulted in less exposure to nature, climate, and sunshine, supporting the hygiene hypothesis and hypovitaminosis.?

The gut microbiome is a unique, highly-active organ, responsible for performing many beneficial physiologic and immunologic functions. A number of mucosal surfaces in the human body are inhabited by microbiota including the lungs, surface of the eye, mouth, and gut, but the gut is the largest and probably most critical to the homeostatic balance of the immune system. The gut-associated lymphoid tissue (GALT) is the largest immune reservoir in the human body, representing approximately $80 \%$ of the immune compartment. ${ }^{4}$ Every mucosal surface is colonized by microflora unique to that site and is distinct. Elie Metchnikov, who was awarded the Nobel Prize in Medicine in 1908, first postulated the importance of the gut microbiome in aging and advocated the use of "probiotics" to resolve aging and human disease. Louis Pasteur, the renowned French microbiologist speculated that life would be impossible in the absence of microbes. However, it was not until almost a century later that the microbiome was defined, by the Nobel laureate Joshua Lederberg in 2001, as the interaction of the genetic material comprising the microbial flora that colonizes a mucosal surface, with the genome of the host. ${ }^{8}$

The gut microflora is comprised of over 100 trillion bacteria, as well as viruses, archaea (an ancient single-celled microorganism without a nucleus or organelles), and fungi. The microbiome is a remarkably complex ecosystem with many bacterial phyla and perhaps as many as 5,000 different strains of just bacteria alone. Every human, as with all living organisms (plant or animal), has a unique, individual microflora similar to a fingerprint for which there is considerable overlap. There is a bidirectional interaction between the genome of the colonized microbial flora of the host mucosal surface and the genome of the host that facilitates the dynamic and vital function of this organ. Interactions between the microbiota and the CNS, known as the gut-brain axis, may occur at interconnected pathways including the immune, neural, and endocrine systems. In addition to its role in maintaining normal bowel-related functions, the gut microbiome has an immunological role, and appears implicated in both metabolic and immune-mediated disorders. ${ }^{2,4} \mathrm{~A}$ number of factors affect the initial colonization of the gut at the time of birth including the mode of delivery, gestational age, diet, hygiene, and antibiotics. ${ }^{9}$ The commensal microbiota (the micro-organisms and viruses associated with the human gastrointestinal tract) outnumber human cells by at least 10-fold and encode more than 100-fold more unique genes than the host genome-Firmicutes and Bacteroidetes are the dominant phyla in the gut of the healthy mammalian body. ${ }^{10,11}$ Vertebrates and bacteria have evolved together, and normal functioning of the digestive and immune system depends on the presence of non-pathologic, symbiotic bacteria. ${ }^{2}$

In 1989 Strachan proposed the "hygiene hypothesis", which suggests that when people experience lower exposure to microbes, they are more likely to develop autoimmune disorders such as allergy and MS.,12 Thus the cleaner our immediate environment, the more we see a reduction in infectious diseases but a substantial increase in autoimmunity. Pedrini suggested that there may be a relationship between Helicobacter pylori infection and MS in females- $H$. pylori seropositivity and MS risk was almost exclusively seen in females. This result could indicate a protective role for $\mathrm{H}$. pylori, but these findings need to be further substantiated..$^{13}$ Most studies on the microbiome and MS have focused on the two major bacterial phyla (Bacteroides and Firmicutes) and archaea. ${ }^{11}$ The National Institutes of Health (NIH)-sponsored Human Microbiome Project aimed to catalog the microbial flora of healthy individuals. Findings to date strongly suggest that the microbiome plays a major role in both the health and disease of the host." Here, we review the role of the microbiome in host immunity and in the pathogenesis and treatment of MS.

\section{The microbiome and immunity}

Findings from the Human Microbiome Project indicate that a healthy gut microbiota is exemplified by its diversity and resilience. ${ }^{11}$ There are a number of ways in which the gut microbiota contributes to health including:

- maintaining an intact intestinal barrier;

- inhibiting pathogenic organisms from colonization;

- regulating host physiology;

- regulating host immune responses, by keeping a balance between pro- and anti-inflammatory responses.

Changes to the gut microbiota, and subsequent changes in its metabolic network, disturb the immune homeostasis resulting in possible intestinal and systemic disorders. As noted, the gut is the major immune organ of humans and other mammals comprising over $80 \%$ of the known lymphatic tissue. The immune system plays both a potent inflammatory role as well as highly critical anti-inflammatory processes. Current data would suggest that the major inflammatory mechanism in experimental autoimmune encephalomyelitis (EAE), as well as human MS, is the recruitment of T-helper 17 (Th17) cells to the sites of inflammation within the CNS wherein interleukin (IL)-17 is expressed and secreted, mustering a potent inflammatory response. This inflammatory response is countered by the induction and recruitment of immune regulatory cells, in particular T and B cells.

Most studies to date have focused on the importance of $\mathrm{T}$ regulatory (Treg) cells in controlling the IL-17-driven inflammatory response. The Treg cells are a subset of $\mathrm{T}$ Iymphocytes that primarily function to modulate and suppress the immune system and reduce pathological self-reactivity as seen in MS and other autoimmune conditions. ${ }^{14}$ The gut microbiota has a crucial role in the development of the immune system. ${ }^{9}$ The gut is the reservoir of both the highly-inflammatory Th17 cells as well as the Treg- and B regulatory (Breg)-cell populations. Commensal bacteria are involved in the production of both IL-10-producing Treg and Breg cells, whereas others make possible the maturation of Th17 cells. Although not absolute, in general, the Bacteroides population is responsible for the induction of the Treg cells, whereas Firmicutes drive Th17. It is likely that these Treg cells may help to suppress the immune system response to commensal bacteria, whereas Th17 cells could have a role in preparing for attack by pathogens. ${ }^{9}$ In addition, gut microbiota have an effect on dendritic cells, natural killer (NK) cells, T cells and B cells, and can have beneficial effects on the immune system. ${ }^{9}$ Gut microbiota also have metabolic functions including salvage of dietary sugars, production of short-chain fatty acids (SCFAs), deconjugation of bile acids and drug metabolism. SCFAs are produced by bacteria in the colon from fermenting non-digestible carbohydrates (e.g. pectins, hemicelluloses, gums). Butyrate, a SCFA, is an important fuel source for colonic epithelium and has profound modulator effects on the immune system: 
- enhances IL-10 and IL-4 production from CD3-stimulated monocytes, which favors non-inflammation;

- reduces the adherence of leukocytes to the vascular endothelium, resulting in apoptosis of $T$ cells and inhibition of interferon gamma (IFN- $\gamma$ ) signal transducer and activator of transcription 1 signaling axis;

- up-regulates populations of colonic Treg cells via signaling through a G-protein-coupled receptor for SCFAs;

- may promote remyelination, microglia function and oligodendrocyte differentiation. ${ }^{15}$

Gut microbiota promote the differentiation of Breg cells in the spleen and mesenteric lymph nodes, which can reduce excessive immune responses through the release of IL-10. Experiments have shown that changes in the gut microbiota following treatment with antibiotics or changes in the sterility of housing conditions reduced the number and function of Breg cells in mice. ${ }^{16}$ Such Breg cells have been shown to ameliorate T-cell-mediated colitis in mice by inducing Treg-1 cells via an IL-27-dependent mechanism. ${ }^{17}$

\section{The gut microbiome and neurological conditions}

Differences in the gut microbiome have also been described in other autoimmune disorders that affect the CNS, such as neuromyelitis optica (NMO), in which certain bacteria, especially Clostridium perfringens, were found to be more abundant when compared to patients with MS and to healthy controls. ${ }^{18} \mathrm{C}$. perfringens was the second most significantly enriched taxon and the species most highly associated with NMO. Since certain Clostridia species (possibly $C$. perfringens) in the gut have been implicated in the regulation of the balance between Treg cells and Th17 cells, they may contribute to the pathogenesis of NMO. ${ }^{18}$ Furthermore, dysbiosis of the microbiome has been implicated in numerous diseases unrelated to the CNS, including diabetes, obesity, cancer, and inflammatory bowel disease (ulcerative colitis, Crohn's disease). ${ }^{19}$ There is also preliminary evidence that the gut microbiome is associated with other neurological conditions including Alzheimer's disease, Parkinson's disease, major depressive disorder, schizophrenia, spinal cord injury, stroke, and autism spectrum disorder.20

\section{The gut microbiome and multiple sclerosis Changing the gut microbiome to enhance therapeutic benefit}

The International MS Microbiome study (iMSMS) has been established with the goal of characterizing the gut microbiome in patients with MS. ${ }^{21,22}$ This initiative includes research teams from three continents and aims to analyze stool and DNA samples from 2,000 subjects with all subtypes of MS and the same number of matched, healthy controls. Many of the subjects are either off therapy or were never treated. Both immunoprofiling and transfer of fecal microbiota into germ-free mice will be undertaken in order to study the potential mechanisms of disease pathogenesis induced by gut dysbiosis. This technique was used by Berer et al. and demonstrated that MS-derived microbiota can cause an MS-like autoimmune disease in transgenic mice. ${ }^{23}$ Other aims include investigating the potential consequences for disease pathogenesis and therapeutic interventions through modification of the gut microbiota with probiotics, prebiotics, antibiotics, fecal transplants, or a combination of these. Probiotics are products that contain an adequate dose of live microbes that have been documented in target-host studies to confer a health benefit.. ${ }^{24} \mathrm{~A}$ prebiotic is a substance that is selectively utilized by host microorganisms conferring a health benefit. ${ }^{25} \mathrm{~A}$ fecal transplant is the administration of a solution of fecal matter from a donor into the intestinal tract of a recipient in order to directly change the recipient's gut microbial composition and confer a health benefit. ${ }^{26}$ Although fecal transplant has been of great benefit in C. difficile membranous colitis it has had uncertain success in inflammatory bowel disease such as Crohn's disease. ${ }^{27,28}$ The effectiveness of this procedure in MS may face the same dilemma and a fecal transplant may not have durability once the gut microflora profile has been established usually by age $3-5$ years. A number of small studies and case reports are evaluating the potential benefit of fecal transplantation in patients with MS (e.g. ClinicalTrials.gov identifer NCT03183869). For example, a case study of a patient with secondary progressive MS (SPMS) given a single fecal microbiota transplant resulted in immediate stabilization of Expanded Disability Status Scale score and a slight improvement in functional scores over 10 years. ${ }^{29}$

\section{Gut microbiome studies in animal models of multiple sclerosis}

Numerous studies examining the role of the gut microbiota in animal models of EAE have been reported. ${ }^{4}$ These include germ-free housing conditions, treatment with antibiotics, orally administering Bacteroides fragilis wild type or the single polysaccharide capsular antigen (PSA) from B. fragilis, oral treatment with Salmonella typhimurium expressing colonization factor antigen I (CFA/I) fimbriae from Escherichia coli, oral administration of Bifidobacterium animalis, oral administration of Lactobacillus species alone or with other lactic acid bacteria or Bifidobacterium bifidum and Streptococcus thermophilus, and oral administration of heat shock protein 65 (Hsp65)-producing Lactobaccilus lactis. All of these treatments decreased the EAE clinical score.

\section{Animal models}

It has been demonstrated that in EAE models, there is a correlation between disease resistance and/or susceptibility to alterations in both the diversity and abundance of gut commensals referred to as alpha and beta. This resistance occurs by the induction of Treg and Breg cells from the GALT. In contrast, disease progression and inflammatory activity is associated with the induction of IL-17 T cells. ${ }^{30}$ In EAE models, numerous experiments have demonstrated that alteration of the gut microbiota results in both effector and regulatory phases of CNS demyelination including MS. ${ }^{31}$ Furthermore, preliminary investigations in humans indicate that gut dysbiosis may have an effect on disease progression. Ochoa-Repáraz et al. suggest that the gut microbiome is an important organ in the regulation of tolerance mechanisms and is the major environmental risk factor for CNS demyelinating disease. It follows that amending the gut microbiome may be a safer therapeutic strategy for treating MS. ${ }^{31}$ Furthermore, strategies to amend the gut microbiome could be complementary to disease-modifying therapy (DMTs).

A PSA derived from the human symbiont $B$. fragilis has been shown to induce immune regulation via up-regulation of IL-10 to produce CD39+ Tregs that protect against EAE when adoptively transferred. ${ }^{30}$ Fletcher et al. demonstrated that CD39+ Treg cells can suppress Th17 cells but not IFN- $\gamma$ secreting CD4 ${ }^{+} T$ cells, which may explain the uncontrolled function of these pathogenic T cells in patients with MS. Although, people with relapsing MS have sufficient numbers of CD39+ Tregs in their peripheral blood mononuclear cells (PBMCs), these cells are insufficient in suppressing IL-17 by mitogen stimulated $\mathrm{CD}^{+}{ }^{+}$T cells. ${ }^{32}$ In human PBMCS, PSA can induce phenotypically distinct CD39+, IL-10 producing Tregs, and human Bregs ex vivo. Interestingly, in EAE mice treated with immunomodulatory agents 
(such as glatiramer acetate, teriflunomide, and anti-CD52), upregulation of GALT-derived protective IL-10-producing CD39+ Tregs has been observed, suggesting this $\mathrm{CD}^{+}$population as a potential biomarker for therapeutic efficacy. T cells have also been identified in PBMCs from patients with MS treated with fingolimod or anti-CD52 enhancement of CD39+, indicating a possible common effect of some MS DMTs on the gut microbiome and GALT. ${ }^{28}$

Studies in a T-cell receptor-transgenic spontaneous mouse model (spontaneous EAE) exemplify the important role of the individual gut microbiome-germ-free mice are protected from EAE in the model, but once these germ-free mice are colonized by normal commensal gut bacteria they spontaneously develop EAE. These findings suggest that the autoimmune reactions against CNS tissue are perhaps "remote controlled" by the gut microbiome since they influence GALT, which regulates systemic immunity. ${ }^{33} \mathrm{~A}$ variety of organisms that comprise the gut microflora may either drive inflammation predominantly via IL-17 or are regulatory via induction and secretion of IL-10.

In the non-obese diabetic (NOD) murine model of SPMS (these mice have a biphasic disease that resembles the progression from relapsing-remitting MS [RRMS] to SPMS in humans), mice that developed the severe secondary progressive form of EAE had altered gut microflora (dysbiosis) compared with healthy controls. For example, in severe disease, specific bacteria of the order Clostidiales significantly increased compared with controls, whereas the genus Lactobacillus and an undefined genus of the family Christensenellaceae were reduced. Members of the family Lactobacillaceae promote protection against murine EAE as a result of inducing IL-10-producing Tregs. Furthermore, NOD mice treated with broad spectrum antibiotics or with PSA showed reduced mortality and clinical disease severity versus untreated mice. ${ }^{34}$ This effect is probably due to alterations in immune responses. These and other studies suggest that communication between the host and gut microbiome is bidirectional. Colpitts et al. hypothesized that the gut microbiota is different in acute inflammatory and chronic progressive stages in the NOD mouse modelin their studies, the gut microbiome of NOD mice developing a severe secondary form of EAE had dysbiosis compared with controls. ${ }^{34}$ Also, when treated with a cocktail of broad-spectrum antibiotics mice showed reduced mortality and disease severity versus untreated mice. Antibiotic treatment changed the balance of regulatory and proinflammatory responses in mice and Tregs have a role in the protection resulting from the modulation of the microbiota. These results suggest that changing the microbiome effects CNS inflammatory demyelination. ${ }^{34}$

Jörg investigated the effect of a high-salt diet in EAE mice. Increased sodium concentrations altered the fecal microbiome, notably depleting Lactobacillus murinus. Mice fed the high-salt diet had an exacerbated disease and an altered fecal microbiome compared with controls fed a normal-salt diet and this could be prevented by concomitant treatment with $L$. murinus. Furthermore, mice on a high-salt diet had significantly higher frequencies of Th17 cells in their small intestine than controls, which were reduced by administration of $L$. murinus. In addition, Th17 cells infiltrating spleen and spinal cord were significantly reduced by $L$. murinus administration. These findings suggest that the detrimental effects of high salt are linked to the gut-immune axis. ${ }^{35}$ Although suggested in EAE studies, a well-defined association between high salt intake and clinical MS remains controversial. Results from the BENEFIT study of IFN $\beta-1 \mathrm{~b}$ treatment in patients with clinically isolated syndrome (Betaferon/Betaseron in Newly Emerging Multiple Sclerosis for Initial Treatment; ClinicalTrials.gov identifer NCT00185211) indicated that sodium levels were not associated with conversion to clinically definite MS over 5 years. ${ }^{36}$ Alternatively, Sumida et al. demonstrated an imbalance of the Treg cytokines IFN- $\gamma$ and IL-10 in patients with MS with high salt intake, suggesting an immunologic link between environmental factors and clinical disease activity. ${ }^{37}$

\section{Multiple sclerosis disease-modifying therapies and the microbiome}

Studies of MS DMTs have also suggested a potential role of the microbiome. Castillo-Álvarez et al. investigated the gut microbiota composition in 30 patients with MS either treated with IFN $\beta$-1b or not, and 14 healthy controls. Significant differences in Firmicutes, Actinobacteria, and Lentisphaerae, and six bacteria species were observed between controls and untreated patients but these differences were not seen when treated patients were compared with controls. Furthermore, the number of Prevotella copri were significantly reduced in untreated patients compared with controls, but levels were significantly higher in treated patients than in those untreated with the number similar to that found in controls. Thus, improvement in patients treated with IFN $\beta$-1b may be a result of its effect on the gut microbiota. ${ }^{38}$

In order to investigate the effects of teriflunomide on the GALT immune cells in mice, B6 mice were treated daily by oral gavage with $20 \mathrm{mg} / \mathrm{kg}$ teriflunomide for 4 weeks. Treatment reduced the percentage of $\mathrm{T}$ cells, B cells, dendritic cells, and monocytes/macrophages of Peyer's patches, with a significant increase in the frequency of $\mathrm{CD} 39^{+}$Tregs compared with controls. It is therefore possible that specific gut CD39+ Tregs contribute to the efficacy of teriflunomide. ${ }^{39}$ Similarly, mice treated orally with teriflunomide showed a decrease in the percentages of antigen-presenting cells of the Peyer's patches compared with controls, but a significant increase in the relative frequency of CD39+ Treg cells..$^{40}$ Furthermore, the protective effect of GALT-derived teriflunomide-induced CD39+ Treg cells was confirmed by the adopted transfer into recipient EAE mice. These findings indicate that specific GALT-derived CD39+ Treg cells may contribute to the efficacy of teriflunomide in relapsing MS. ${ }^{41}$ In addition, the CD39+ population has been shown to be increased in mice with EAE and in patients with MS treated with anti-CD52 (alemtuzumab). Furthermore, a study of patients with RRMS treated with glatiramer acetate, dimethyl fumarate, or untreated showed some bacterial genera were differentially regulated in treated patients and common bacterial groups were reduced with treatment. ${ }^{42}$

In animal models of MS, altering the gut microbiome results in both the effector and regulatory phases of CNS demyelination. Preliminary studies in humans with MS suggest that dysbiosis in the gut microbiome may affect progression of disease, and the gut microbiome can be considered the key organ for regulating tolerance mechanisms and the major environmental risk factor for CNS demyelinating disease (Figure 1). ${ }^{31}$

\section{Gut microbiome studies in humans}

It is estimated that over 5,000 strains of bacteria may exist in the human gut comprising a complex ecosystem. Many groups have studied the gut microbiome and findings include an increase in some phyla and a decrease in others, changes in the metabolome in patients with MS and healthy controls, and changes after the administration of the probiotic VSL\#3 


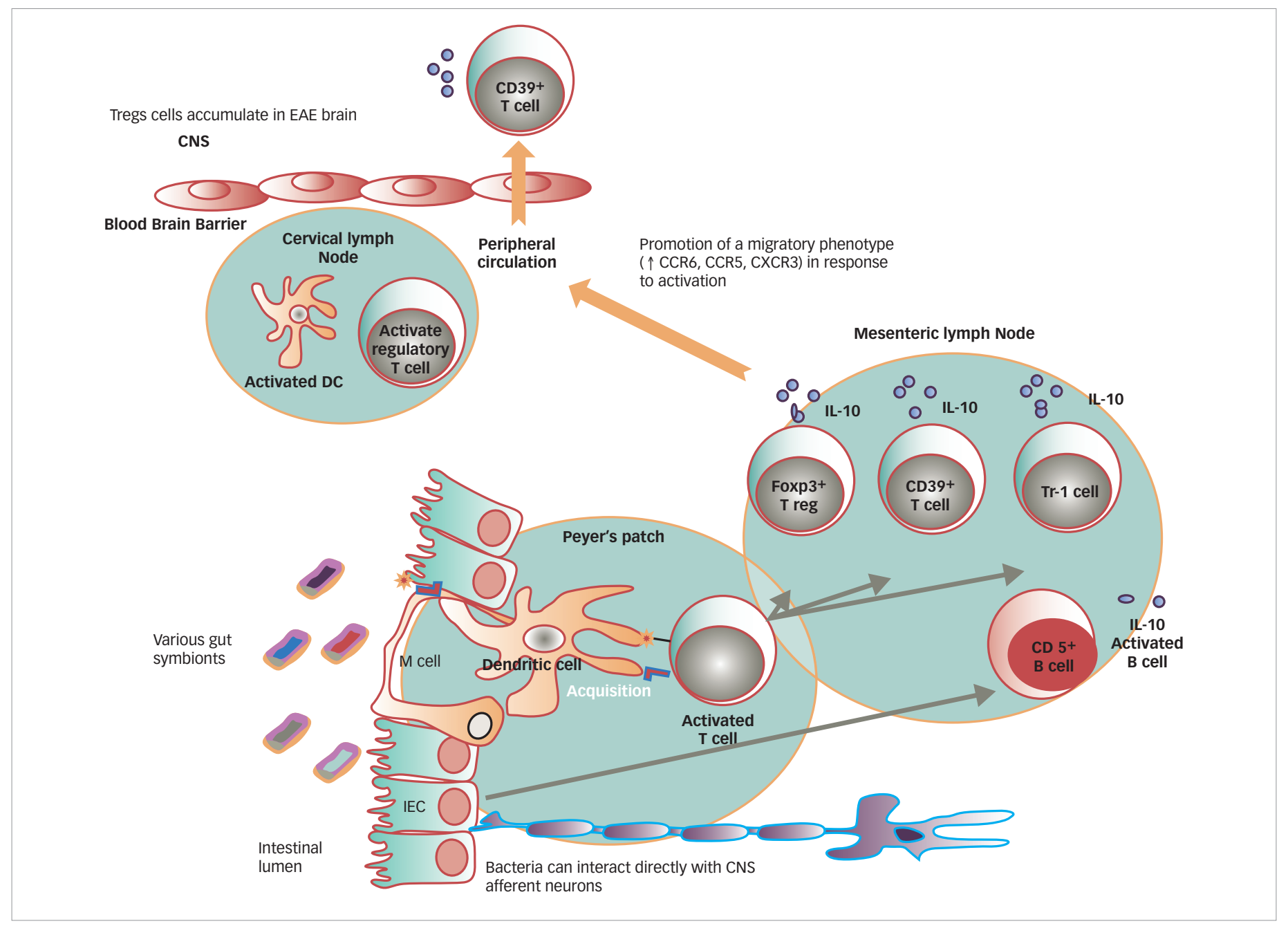

CCR5/6 and CXCR3 = chemokine receptors; $C N S=$ central nervous system; $D C=$ dendritic cell; EAE = experimental autoimmune encephalomyelitis; Foxp3 = transcription regulator Fork-head box P3; IEC = intraepithelial cells; IL-10 = interleukin 10; $\mathrm{M}$ cell = microfold cell; Tr-1 cell = type 1 regulatory $T$ cell; Treg = Tregulatory cell. Reproduced by permission from L Kasper and $\mathrm{J}$ Ochoa (unpublished).

(Alfasigma, Covington, LA, USA) in patients with RRMS. Table 1 summarizes the changes in microbial abundance in patients with MS. ${ }^{11,23,42-50}$

Although patients with MS seem to have a virtually normal level of circulating Treg cells, these cells appear to be dysfunctional as they are unable to adequately suppress both the proliferation of proinflammatory cells and the secretion of proinflammatory cytokines (e.g. IFN- $\gamma$ and IL-17). Since the reservoir for Th17 cells that secrete IL-17, and Treg cells that express the transcription regulator Fork-head box p3 (Foxp3), is located in the GALT, its role within the microbiome could be critical to the understanding of immunopathogenesis and regulation in CNS demyelinating disease. ${ }^{51}$ A number of studies provide evidence that MS affects gut microbiota composition, resulting in changes to the relative abundance of specific bacteria and archaea species. Some bacterial species that promote immunosuppressive Tregs are decreased in the gut of patients with MS and the function of a phenotype of Tregs expressing CD39 seems to be reduced. ${ }^{51}$

Jangi and colleagues investigated the gut microbiota in 60 patients with MS and 43 healthy controls. ${ }^{52}$ Increases in Methanobrevibacter and
Akkermansia, together with decreases in Butyricimonas, were identified in patients with MS and these changes correlated with variations in the expression of genes involved in dendritic cell maturation, IFN signaling and NF-kB signaling pathways in circulating T cells and monocytes. Interestingly, there was an increase in Prevotella and Sutterella and a decrease in Sarcina in patients on DMTs compared with untreated patients. In a different cohort of patients with MS, elevated breath methane was detected compared with controls - this is consistent with the increased gut Methanobrevibacter identified in the first cohort. The study authors commented that these findings indicate that the gut microbiome could have a role in MS pathogenesis, or is a consequence of it, and that further studies are needed to answer these questions. If they show that these microorganisms contribute to or ameliorate MS, new diagnostics and therapies may be developed. ${ }^{52}$

Another study investigated the microbiome of 71 patients with untreated RRMS and 71 healthy controls. ${ }^{45}$ Although major shifts in the bacterial community structure, in terms of alpha or beta diversity, were not evident, there were significant differences in individual microbial taxa 
Table 1: Changes in microbial phyla in patients with multiple sclerosis $11,45,50,52$

\begin{tabular}{|l|l|}
\hline Bacteria (phylum) & Archaea \\
\hline Bacteroidetes (gram-negative anaerobe) & Euryarchaeota \\
Parabacteroides distasonis $\downarrow$ & Methanobrevibacter $\uparrow$ \\
Butyricimonas $\downarrow$ & \\
\hline Proteobacteria (gram-negative aerobe) & \\
Acinetobacter calcoaceticus $\uparrow$ & \\
Hemophilus $\uparrow$ & \\
Mycoplana $\uparrow$ & \\
Pseudomonas $\uparrow$ & \\
\hline Verrucomicrobia (gram-negative anaerobe) & \\
Akkermansia (e.g. Akkermansia muciniphila) $\uparrow$ & \\
\hline Firmicutes (gram-positive anaerobe) & \\
Blautia $\uparrow$ & \\
Dorea $\uparrow$ & \\
\hline
\end{tabular}

$\uparrow=$ increased, $\downarrow=$ decreased. These preliminary findings indicate a possible link between the two major phyla that colonize the human gut and clinical multiple sclerosis (MS). Patients with MS demonstrate a reduction in Bacteroidetes phylum and an increase in Firmicutes phylum. This is important as generally Bacteroidetes are associated with induction of T regulatory cells, whereas Firmicutes with increased interleukin-17 production.

in patients with MS versus controls. Acinetobacter calcoaceticus and Akkermansia muciniphila were significantly increased compared with controls and induced proinflammatory responses both in human PBMCs and monocolonized mice. In contrast, Parabacteroides distasonis were the most significantly reduced bacteria in patients with MS and were found to stimulate anti-inflammatory IL-10-expressing human $\mathrm{CD} 4^{+} \mathrm{CD} 25^{+} \mathrm{T}$ cells and IL-10+ Foxp3 ${ }^{+}$Tregs when inoculated in mice. Furthermore, when microbiota were transferred from patients with MS to germ-free mice, more severe EAE symptoms and reduced proportions of $\mathrm{IL}-10^{+}$Tregs were observed compared with microbiota from healthy controls. The bacteria associated with MS were shown to regulate T lymphocyte mediated adaptive immune response and also contributed to the proinflammatory environment in vitro and in vivo. Thus, specific gut bacteria have been identified that regulate adaptive autoimmune responses. ${ }^{45}$

The gut microbial composition in 34 monozygotic twins discordant for MS has been investigated to identify any differences in the microbiome. ${ }^{23}$ No major differences were seen in the overall microbes but there were significant increases in some taxa, for example Akkermansia in the untreated twins with MS. In addition, a significantly higher incidence of autoimmunity occurred when the microbiota of the twins with MS were transplanted into a transgenic mouse model of spontaneous brain autoimmunity compared with the healthy twin. The microbiota of the colonized mice consistently showed a number of differences including the presence of Sutterella, which induces a protective immunoregulatory profile in vitro. Compared with mice transplanted with samples from the healthy twins, those receiving microbes from the twins with MS had immune cells that produced less IL-10, which may have a regulatory role in spontaneous CNS autoimmunity since mice colonized with healthy-twin fecal material had increased disease incidence. Thus, microbiota from patients with MS appear to have factors that precipitate an MS-like autoimmune disease in these transgenic mice. ${ }^{23}$

A further study in patients with RRMS and healthy controls ( $n=31$ and $\mathrm{n}=36$ respectively) confirmed that certain genera showed an increased abundance of microorganisms (Pseudomonas, Mycoplana, Haemophilus,
Blautia, Dorea) in patients with MS whereas other genera were increased in controls (Parabacteroides, Adlercreutzia, Prevotella). These results support the hypotheses that patients with MS have gut dysbiosis. ${ }^{44}$ Several publications have also reported that patients with MS have gut microbial dysbiosis with depletion and enrichment of some genera compared with healthy controls. ${ }^{44,50,52}$ The overall conclusions from these studies are that the microbiome from patients with MS shows a reduction in bacteria that induce immunoregulatory cells and an increase in those that induce proinflammatory responses. ${ }^{11}$

It is not understood which of these two changes is responsible for disease pathogenesis. Most clinical immunologic data indicate that patients with MS and age-matched controls have similar patterns of inflammatory cells in their circulation. To the contrary, it appears that the Treg and possibly B-cell population in those with MS is insufficient in IL-10 production and unable to suppress IL-17 mediated inflammatory response.

In order to investigate whether the gut microbiota contribute to the pathogenesis of MS, the functional properties of gut microbiota have been characterized from 64 treatment-naive patients with MS and 68 healthy controls. ${ }^{53}$ Certain individual microbial taxa were significantly associated with MS. In vitro assays to characterize the functional properties of the MS gut microbiota demonstrated that Acinetobacter calcoaceticus from patients with MS reduced Treg differentiation and increased Th1 and Th2 differentiation. Furthermore, Akkermansia muciniphils (which was more abundant in MS samples) recapitulated Th1 lymphocyte expansion. However, Parabacteroides distasonis was significantly reduced in patients with MS and stimulated $\mathrm{CD}^{+}{ }^{+}$T-lymphocyte differentiation into a CD25+ IL-10+ regulatory phenotype. ${ }^{53}$ In a similar investigation, the abundance of A. calcoaceticus differed between patients with MS and controls, and more gut bacteria from patients with MS were bound to immunoglobulin G, which indicates that a larger proportion might escape the gut lumen, enter the circulation and illicit an autoimmune response in these patients. ${ }^{54}$

\section{Secondary progressive multiple sclerosis}

In a preliminary study, Eleftheriou demonstrated that Bacteroides finegoldii, Eubacterium ramulus and Ruminococcus lactaris occurred in a statistically significant higher abundance in patients with SPMS with active disease; whereas Sutterella wadsworthensis abundance was greater in patients with stable SPMS compared with controls. ${ }^{49}$ As with relapsing disease, causation has yet to be established.

\section{Clinically isolated syndrome}

The composition of the microbiome has been studied in 20 patients with clinically isolated syndrome and 20 healthy controls. There was a lower abundance of Bacteroides and decrease in species richness in patients with clinically isolated syndrome compared with healthy controls. In addition, patients with clinically isolated syndrome were found to have an increase in pathogenic Th17 cells expressing Toll-like receptor 2, and a decrease in Treg cells producing IL-10 and expressing CD39.50

\section{Probiotics and poly-biotics as adjunctive management of multiple sclerosis}

It is likely that dietary factors could ameliorate or exacerbate MS symptoms in RRMS and primary progressive MS by controlling metabolic and inflammatory pathways in cells and altering the commensal organisms in gut microbiota. The main environmental factors associated with MS, such 
as geographical distribution, low vitamin $\mathrm{D}$, and high body mass index can all be linked to diet and lifestyle. ${ }^{55}$ High-calorie diets typical in the west (high intake of salt, animal fat, red meat, fried foods) together with limited exercise, up-regulate the metabolism of cells towards biosynthetic pathways such as those of proinflammatory molecules that cause adverse effects-altering immunity in the intestine and contributing to low-grade systemic inflammation and dysbiosis. In contrast, diets with lower calories (vegetables, fruit and legumes) together with exercise have a beneficial effect on nuclear receptors and enzymes involved in the up-regulation of oxidative metabolism, down-regulation of proinflammatory molecules synthesis, and restoring or maintaining a healthy gut microbiota. ${ }^{55}$

The probiotic VSL\#3 comprises eight bacteria and has been shown to induce $\mathrm{IL}-10^{+}$and IL-10-dependent transforming growth factor beta (TGF- $\beta$ )-bearing regulatory cells in the gut of a mouse model of colitis. It also promotes neuroprotection in a mouse traumatic spinal cord injury model and is beneficial in ulcerative colitis and diabetes. ${ }^{47}$ In a study of patients with RRMS, either untreated $(n=2)$ or on glatiramer acetate $(G A ; n=7)$, and healthy controls ( $n=13)$, VSL\#3 (3,600 billion colony-forming units [CFU]/day) was administered twice daily for 2 months. ${ }^{47}$ Healthy controls had decreased alpha diversity (ecological diversity in a given sample/type of microbial species) whereas there was no change in patients with RRMS. Several species including Lactobacillus, Streptococcus and Bifidobacterium increased in all patients. In addition, an anti-inflammatory peripheral immune response characterized by lower frequency of intermediate monocytes $\left(\mathrm{CD} 14^{\text {high }} \mathrm{CD} 16^{\text {low }}\right)$ in patients with MS, and a trend towards decreased frequency of inflammatory monocytes $\left(C D 14^{\text {low }} C D^{\text {high }}\right)$ in controls, was observed. Patients with MS also had decreased mean fluorescence intensity of CD80 on classical monocytes and decreased human leukocyte antigen-antigen $D$ related (HLA-DR) on dendritic cells. These results suggest that administration of VSL\#3 induces changes in the composition of the gut microbiota associated with an anti-inflammatory peripheral innate immune response. ${ }^{47}$

\section{Probiotics and poly-biotics as adjunctive management of other central nervous system disorders}

The role of probiotics has also been studied in animal models of other CNS disorders. A 2011 study investigated neurotransmitter receptors in the CNS of normal, healthy animals. Gamma-aminobutyric acid (GABA) is the main CNS inhibitory neurotransmitter involved in regulating many physiological and psychological processes, and changes in its receptor expression have been implicated in the pathogenesis of anxiety and depression. ${ }^{56}$ It was found that mice fed the probiotic Lactobacillus rhamnosus had significant induced changes in the levels of $\mathrm{GABA}_{\mathrm{B} 1 \mathrm{~b}}$ mRNA in the brain with increases in cortical regions and decreases in the hippocampus, amygdala, and locus coeruleus compared with controls (fed broth). Lower levels of stress-induced corticosterone and anxiety/depressive behavior were seen compared with control mice.56

\section{Effect of probiotics and poly-biotics in healthy human subjects}

David and coworkers examined the effects of short-term changes to diet on the microbiome in healthy humans..$^{57} \mathrm{~A}$ plant-based diet rich in grains, legumes, fruits, and vegetables was compared with an animal-based diet which included meats, eggs, and cheeses consumed ad libitum for 5 consecutive days by six male and four female volunteers. Each diet change rapidly altered the gut microbiota 1 day after the diet reached the distal gut microbiota. The abundance of bile-tolerant microorganisms (Alistipes, Bilophila and Bacteroides) increased, while Firmicutes (Roseburia, Eubacterium rectale and Ruminococcus bromii) that metabolize plant polysaccharides, decreased in the animal-based diet. ${ }^{57}$

Overall, the effect of probiotics and poly-biotics is extremely complex. For example, most species of Firmicutes are associated with IL-17 and proinflammation and there is a need to clarify this disparity between pro- and anti-inflammatory activities. It must be noted that each of the major phyla has hundreds of different species and strains. Within each phylum may be found bacterial or bacterial antigens that have both inflammatory and anti-inflammatory properties. We are at the very tip of an iceberg in understanding the complexity of the microbiome in the control of the immune response.

\section{Treatment/modification of the microbiome Diet and the microbiome}

One of the most significant changes in human biology over the last 200 years has been in relation to diet, which has been shown to have an important effect on the microbiome. Experiments in germ-free C57BL/6J mice transplanted with human adult fecal microbial communities showed that switching from a low-fat, plant polysaccharide-rich diet to a high-fat/high-sugar 'Western' diet altered the microbiota composition in a single day. Both metabolic pathways and gene expression changed. ${ }^{14}$

In order to investigate the effect of diet, three calorie restriction diets (intermittent diet of $75 \%$ reduction in calorie needs 2 days/week and $100 \%$ of daily needs intake 5 days/week; continuous calorie restriction diet [22\% daily reduction in energy needs]; and a weight-stable diet [100\% of daily calorie needs]) were studied in 36 patients with MS over an 8-week period. All patients lost weight and had significant changes to the circulating metabolome. ${ }^{43}$ Furthermore, a cross-sectional survey in participants in the North American Research Committee on MS (NARCOMS) suggested that a healthy diet and lifestyle are associated with lower disability and symptom burden in MS. ${ }^{58}$ Differences in these diets on the composition and colonization patterns of the human microbiome in those with MS has yet to be elucidated.

\section{Therapeutic intervention of the microbiome}

There has been emerging evidence that MS DMTs affect gut microbiota composition. A small sub-study examined the microbiota of seven vitamin D insufficient patients with RRMS (five of which were treated with GA, but three did not submit stool samples before and after supplementation-four patients with MS, of which two were treated, gave both samples) and eight healthy controls. The abundance of some bacteria (e.g. Faecalibacterium) was lower in patients with MS, and GA-treated patients had differences compared with untreated patients (including Bacteroidaceae, Faecalibacterium, Ruminococcus). Furthermore, vitamin D supplementation increased Akkermansia, Faecalibacterium and Coprococcus in untreated patients with MS compared with other groups. It may be concluded that although bacterial genera were similar, some taxa were different in MS or control subjects. ${ }^{59}$

Another study assessed whether current immunomodulatory drugs for RRMS differentially impacted the composition of the microbiota. In patients with RRMS, either untreated or treated with GA or dimethyl fumarate for at least 3 months, several bacterial genera were regulated with treatment compared with untreated patients. Of note, GA rebalanced microbial 
abundance (equal number down- or up-regulated) while dimethyl fumarate reduced most of the bacteria that were changed. Both treatments reduced a common group of bacteria, suggesting a possible contribution to the efficacy of these DMTs. ${ }^{42}$

Kasper et al. investigated the effect of PSA on the differentiation of Treg cells derived from naïve T cells isolated from patients with MS PBMCs were isolated from patients with MS and healthy controls (both $n=18)$. There was a significant increase in the frequency of Foxp3 ${ }^{+} T$ cells when $T$ cells from healthy controls were cultured with PSA, and this also occurred with T cells isolated from untreated or treated patients with MS. Additionally, in naïve T cells from patients treated with GA, PSA induced Treg conversion. Thus, a gut-derived commensal antigen can amplify a Treg-cell response in MS. ${ }^{48,60}$

\section{Conclusions}

In the past decade, we have come to appreciate and understand that the microbiome is an important organ within all living creatures. For mammals, studies of the microbiome have become an important source for novel information as to how the mammalian body is under the physiologic and immunologic control of the microbiome, and in particular, the gut microbiome which is the largest mucosal immune surface. The gut contains trillions of bacteria, viruses, and fungi containing millions of genes distinct from their host. These genes produce copious numbers of metabolites, many of which pass through the lumen of the gut and create the gut-brain axis that is involved in a wide range of physiologic and immunologic functions. Disruption of the normal homeostatic balance of these microflora leads to dysbiosis and various disease states including autoimmunity such as occurs in human MS.

As such, the microbiome can be considered a "treasure trove" of potential therapeutics for treating human disease. Accordingly, there has been a rapidly expanding interest, in both academia and industry, to resource this unique organ of which we know very little about. This interest goes well beyond classic immune-medicated conditions and includes: cerebrovascular disease; atherosclerosis; physiologic and endocrinologic conditions; as well as a broad band of neurologic conditions including Parkinson's disease, Alzheimer's disease; and affective disorders such as autism, depression, and psychosis to name a few. In regard to the immune system and CNS demyelination, the gut microbiota plays a central role in the development of the immune system, with the GALT being the largest immune reservoir in humans. There is a bidirectional interaction between the microflora that colonize the gut and other mucosal surfaces, and the
Figure 2: The gut microbiome is associated with many human conditions

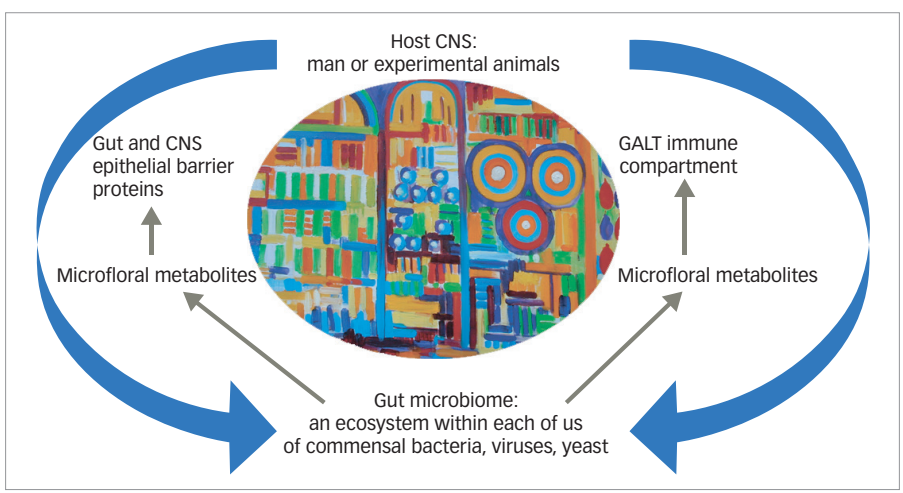

The gut microbiome is a bidirectional organ associated with a variety of human conditions including experimental neurologic diseases such as multiple sclerosis, autism, Parkinson's disease, Alzheimer's disease, depression, and stroke. CNS = central nervous system; GALT = gut-associated lymphoid tissue. Reproduced by permission from L Kasper (unpublished)

brain, giving rise to a gut-brain axis that may be involved in many of the neurologic diseases for which an etiology is yet to be determined (Figure 2). We are on the forefront of understanding for a new wave of human biology that may lead to a wide range of therapeutic interventions that will revitalize the dysbiotic mucosal surface. This may be through pro- and prebiotics, antibiotics (due to alterations in immune responses), recolonization with normal gut flora (via fecal or combination of known symbionts), or ultimately the identification of specific commensal bacteria that regulate the immune system. Pursuit of single antigenic molecules, such as the studies on $B$. fragilis-derived PSA, may offer therapeutic advantage by readjusting the gut flora in such a way that the afflicted patient may be able to control disease progression without the potent agents that are currently on the forefront of MS therapy; they may also be used adjunctively.

As our information base regarding the dysbiotic state in MS evolves, a path to better and safer therapeutic interventions will arise. The opportunity to treat a human condition such as MS with therapies that do not destroy or significantly and irreversibly alter the immune system will set a new standard for treatment. Alteration of dietary habits in association with other non-invasive techniques will allow for the manipulation of the gut bacteria and reduce the dysbiosis associated with the disease state. The future of human biology may be in understanding how to readjust the trillions of microflora within each of us that will lead to better health. $\square$
1. Rejdak K, Jackson S, Giovannoni G. Multiple sclerosis: a practical overview for clinicians. British Medical Bulletin. 2010:95:79-104. . Joscelyn J, Kasper LH. Digesting the emerging role for the gut microbiome in central nervous system demyelination. Mult Scler. 2014;20:1553-9.

3. Kingwell E, Zhu F, Marrie RA, et al. High incidence and increasing prevalence of multiple sclerosis in British Columbia, Canada: findings from over two decades (1991-2010). J Neurol. 2015;262:2352-63.

4. Mielcarz DW, Kasper LH. The gut microbiome in multiple sclerosis. Curr Treat Options Neurol. 2015:17:344.

5. Ascherio A, Munger KL, Lünemann JD. The initiation and prevention of multiple sclerosis. Nat Rev Neurol. 2012;8:602-12.

6. Ochoa-Repáraz J, Kasper LH. Gut microbiome and the risk factors in central nervous system autoimmunity. FEBS Lett. 2014;588:4214-22.

7. Pierrot-Deseilligny C, Souberbielle J-C. Is hypovitaminosis D one of the environmental risk factors for multiple sclerosis? Brain. 2010;133:1869-88.

8. Lederberg J, McCray AT. 'Ome Sweet 'Omics - A genealogical treasury of words. Scientist. 2001;15:8.
9. Bhargava P, Mowry EM. Gut microbiome and multiple sclerosis Curr Neurol Neurosci Rep. 2014;14:492.

10. Belkaid $Y$, Naik $S$. Compartmentalized and systemic control of tissue immunity by commensals. Nat Immunol. 2013;14:464-53.

11. Shahi SK, Freedman SN, Mangalam AK. Gut microbiome in multiple sclerosis: the players involved and the roles they play. Gut Microbes. 2017;8:607-15.

12. Strachan DP. Hay fever, hygiene, and household size. BM 1989;299:1259-60.

13. Pedrini MJ, Seewann A, Bennett KA, et al. Helicobacter pylori infection as a protective factor against multiple sclerosis risk in females. J Neurol Neurosurg Psychiatry. 2015;86:603-7.

14. Turnbaugh PJ, Ridaura VK, Faith JJ, et al. The effect of diet on the human gut microbiome: a metagenomic analysis in humanized gnotobiotic mice. Sci Trans/ Med. 2009;1:6ra14.

15. Ochoa-Repáraz J, Kasper LH. The microbiome and neurologic disease: past and future of a 2-way interaction. Neurotherapeutics. 2018;15:1-4.

16. Rosser EC, Oleinika K, Tonon S, et al. Regulatory B cells are induced by gut microbiota-driven interleukin- $1 \beta$ and interleukin- 6 production. Nat Med. 2014;20:1334-9.
17. Mishima Y, Liu B, Hansen JJ, et al. Resident bacteria-stimulated interleukin-10-secreting B cells ameliorate T-cell-mediated colitis by inducing T-regulatory-1 cells that require interleukin-27 signaling. Cell Mol Gastroenterol Hepatol. 2015;1:295-310

18. Zamvil SS, Spencer $\mathrm{CM}$, Baranzini $\mathrm{S}$, et al. The gut microbiome in neuromyelitis optica. Neurotherapeutics. 2018;15;92-101.

19. Guinane $\mathrm{CM}$, Cotter PD. Role of the gut microbiota in health and chronic gastrointestinal disease: understanding a hidden metabolic organ. Ther Adv Gastroenterol. 2013;6:295-308.

20. Sharon G, Sampson TR, Geschwind DH, et al. The central nervous system and the gut microbiome. Cell. 2016;167:915-32.

21. The International MS Microbiome Study (iMSMS). Available at: www.imsms.org (accessed May 18, 2019)

22. Baranzini SE. The International MS Microbiome Study (iMSMS): investigating the role of gut microbiota in multiple sclerosis through open collaboration. ECTRIMS Online Library. 2016;146272: Abstract P432.

23. Berer K, Gerdes LA, Cekanaviciute E, et al. Gut microbiota from multiple sclerosis patients enables spontaneous autoimmune encephalomyelitis in mice. Proc Natl Acad Sci U S A. 
2017;114:10719-24

24. Sanders ME. Probiotics: Definition, sources, selection and uses. Clin Infectious Diseases. 2008;46:S58-61

25. Gibson GR, Hutkins R, Sanders ME, et al. Expert consensus document: the International Scientific Association for Probiotics and Prebiotics (ISAPP) consensus statement on the definition and scope of prebiotics. Nat Rev Gastroenterol Hepatol. 2017:14:491-502

26. Gupta S, Allen-Vercoe E, Petrof EO. Fecal microbiota transplantation in perspective. Ther Adv Gastroentero. 2016:9:229-39.

27. Moayyedi P. Fecal transplantation: any real hope for inflammatory bowel disease? Curr Opin Gastroenterol. 2016;32:282-6.

28. Jeon SR, Chai J, Kim C, Lee $\mathrm{CH}$. Current evidence for the management of inflammatory bowel diseases using fecal microbiota transplantation. Curr Infect Dis Rep. 2018;20:21.

29. Makkawi S, Camara-Lemarroy C, Metz L. Fecal microbiota transplantation associated with 10 years of stability in a patient with SPMS. Neurol Neuroimmunol Neuroinflamm. 2018;5:e459.

30. Kasper L. In the Garden of Good and Evil: the role of the gut microbiome in CNS demyelinating disease. ECTRIMS Online Library. 2017;202520:Abstract 164

31. Ochoa-Repáraz J, Kirby TO, Kasper LH. The gut microbiome and multiple sclerosis. Cold Spring Harb Perspect Med. 2018;8: pii: a029017

32. Fletcher JM, Lonergan $\mathrm{R}$, Costelloe $\mathrm{L}$, et al. CD39+Foxp3 ${ }^{+}$ regulatory $T$ cells suppress pathogenic Th17 cells and are impaired in multiple sclerosis. J Immunol. 2009;183:7202-10.

33. Hohlfeld R. Modulating microbiota: friend or foe? ECTRIMS Online Library. 2015;116742:Abstract 167.

34. Colpitts SL, Kasper EJ, Keever A, et al. A bidirectiona association between the gut microbiota and CNS disease in a biphasic murine model of multiple sclerosis. Gut Microbes. 20172;8:561-73.

35. Jörg S. Salt-sensitive alterations in gut microbiota impact Th17 cells and neuroinflammation. ECTRIMS Online Library. 2017;200148:Abstract P493.

36. Fitzgerald $\mathrm{KC}$, Munger $\mathrm{KL}$, Hartung $\mathrm{HP}$, et al. Sodium intake and multiple sclerosis activity and progression in BENEFIT. Ann Neurol. 2017;82:20-9.

37. Sumida T, Lincoln MR, Ukeje CM, et al. Activated $\beta$-catenin in Foxp $3^{+}$regulatory $\mathrm{T}$ cells links inflammatory environments to autoimmunity. Nat Immunol. 2018;19:1391-402.

38. Castillo-Álvarez F, Pérez-Matute P, Oteo JA, Marzo-Sola ME. The influence of interferon $\beta-1 \mathrm{~b}$ on gut microbiota composition in patients with multiple sclerosis. Neurologia. 2018; pii:S0213-4853(18)30158-0. doi:10.1016/j.nrl.2018.04.006

39. Kircher C, Kasper E, Telesford K, et al. A novel mechanism of action for teriflunomide by the induction of immunosuppressive gut CD39+FoxP3+Tregs in mice. ECTRIMS Online Library. 2015;116035:Abstract P1064.

40. Ochoa-Repáraz J, Kasper E, Kircher C, et al. The gut-brain CD39+ $T$ regulatory cell axis: role of the microbiota and gut associated Iymphoid tissue in regulating inflammatory CNS demyelination. ECTRIMS Online Library. 2015;115883:Abstract P879.

41. Ochoa-Repáraz J, Colpitts SL, Kircher C, et al. Induction of gut regulatory $C D 39^{+} T$ cells by teriflunomide protects against EAE. Neurol Neuroimmunol Neuroinflamm. 2016;3:e291.

42. Casaccia P, Zhu Y, Cekanaviciute E, et al. Effect of oral versus injectable disease-modifying therapies on the epigenomewide DNA methylation and gut microbiota in multiple sclerosis patients. ECTRIMS Online Library. 2016:145837:Abstract P1153.

43. Fitzgerald KC. Calorie restriction diets and changes in the metabolome in people with multiple sclerosis. ECTRIMS Online Library. 2017;200899:Abstract P1244.

44. Jangi S, Gandhi R, Li N, et al. Alterations of the human gut microbiome in multiple sclerosis. ECTRIMS Online Library. 2015;115762: Abstract P406

45. Cekanaviciute $E$, Yoo BB, Runia TF et al. Gut bacteria from multiple sclerosis patients modulate human T cells and exacerbate symptoms in mouse models. Proc Natl Acad SCI U S A. 2017;114:10713-8

46. Chen J, Chia N, Kalari KR, et al. Multiple sclerosis patients have a distinct gut microbiota compared to healthy controls. Sci Rep. 2016;6:28484.

47. Tankou SK, Regev K, Healy BC, et al. Investigation of probiotics in multiple sclerosis. Mult Scler. 2018;24:58-63.
48. Kasper L. CD4+ T cells from multiple sclerosis patients acquire regulatory characteristics following exposure to gut commensal-derived antigen. ECTRIMS Online Library. 2017;200139:Abstract P484.

49. Eleftheriou $\mathrm{E}$. The gut microbiome in active and stable relapsing multiple sclerosis. ECTRIMS Online Library. 2017;200659:Abstract P1004.

50. Rolla S. Gut microbiota alteration in clinically isolated syndrome: a pilot study. ECTRIMS Online Library. 2017;199515:Abstract EP1495.

51. Ochoa-Repáraz J, Kasper LH. The influence of gut-derived CD39 regulatory T cells in CNS demyelinating disease. Transl Res. 2017; 179:126-38.

52. Jangi $S$, Gandhi R, Cox LM, et al. Alterations of the human gut microbiome in multiple sclerosis. Nat Commun. 2016;7:12015.

53. Cekanaviciute E, Debelius JW, Singh S, et al. Gut dysbiosis is a feature of MS and it is characterized by bacteria able to regulate Iymphocyte differentiation in vitro. ECTRIMS Online Library. 2016;147026:Abstract 179

54. Cekanaviciute E, Runia TF, Debelius JW, et al. The influence of microbiota on the adaptive immune response in MS. ECTRIMS Online Library. 2015;116274:Abstract P901.

55. Riccio P, Rossano R. Nutrition facts in multiple sclerosis. ASN Neuro. 2015;7: pii: 1759091414568185.

56. Bravo JA Forsythe P, Chew MV et al. Ingestion of Lactobacillus strain regulates emotional behavior and central GABA receptor expression in a mouse via the vagus nerve. Proc Natl Acad SCi U S A. 2011;108:16050-5.

57. David LA, Maurice CF, Carmody RN, et al. Diet rapidly and reproducibly alters the human gut microbiome. Nature. 2014;505:559-63.

58. Fitzgerald $\mathrm{KC}$, Tyry $\mathrm{T}$, Salter $\mathrm{A}$, et al. Diet quality is associated with disability and symptom severity in multiple sclerosis. Neurology. 2018;90:e1-11.

59. Cantarel BL, Waubant E, Chehoud C, et al. Gut microbiota in MS: possible influence of immunomodulators. I Investig Med. 2015;63:729-34.

60. Burgess JN, Pant AB, Kasper LH, Colpitts Brass S. CD4+ T cells from multiple sclerosis patients respond to a commensal-derived antigen. Ann Clin Trans/ Neurol. 2017;4:825-9. 